\title{
Gobernanza del agua y desarrollo bajo el mercado: las relaciones sociales de control del agua en el marco del Código de Aguas de Chile
}

Governance of water and development within the market: the social relations of control over water in the framework of Chile's Water Code

\author{
Jessica Budds ${ }^{\mathrm{a}}$ \\ Historial del artículo \\ a School of International Development, University of East Anglia, Norwich, Reino Unido. Correo electrónico: j.budds@uea.ac.uk
}

Recibido:

02 de junio de 2020

Revisado

17 de junio de 2020

Aceptado:

22 de junio de 2020

\section{Palabras clave}

Neoliberalismo, orden políticoeconómico, recursos hídricos, relaciones de poder

\section{Keywords}

Neoliberalism, power relations, political-economic order, water resources

\begin{abstract}
Resumen
Mediante la aplicación de principios "neoliberales" (derechos de propiedad privada, mercados y desregulación), el objetivo del Código de Aguas de Chile de 1981 era fomentar la inversión de los usuarios en la infraestructura hidráulica y la eficiencia en el uso del agua. Sin embargo, el Código de Aguas se ve cada vez más cuestionado por su asociación con la sobreexplotación de los cuerpos de agua para obtener ganancias económicas a expensas de las necesidades básicas y la protección ambiental. Muchos análisis han examinado los efectos del Código de Aguas en la gestión del agua, pero han prestado menos atención a su gobernanza, o sea, a las estructuras institucionales, y los procesos de toma de decisiones en torno al recurso hídrico. El objetivo de este artículo es analizar cómo el Código de Aguas ha configurado la gobernanza, cómo esta gobernanza ha influenciado las relaciones sociales de control sobre el agua, y, a su vez, cómo aquellas relaciones sociales han consolidado el orden político-económico del país. Si bien los análisis de la gobernanza, como la práctica de la regulación muestran la naturaleza y el funcionamiento del Código de Aguas, se argumenta que abordar la gobernanza como el proceso de construcción de la regulación revela las relaciones de poder embebidas en el marco y su rol de privilegiar la acumulación capitalista y el poder político. Revelar las relaciones de poder en el diseño, implementación y defensa del Código de Aguas indica las limitaciones de las propuestas de cambio existentes e indica nuevas vías para la transformación hidrosocial.
\end{abstract}

\begin{abstract}
Through the application of "neoliberal" principles (private property rights, markets, and deregulation) the aim of Chile's 1981 Water Code was to foster user investment in water infrastructure and efficiency in water use. However, the Water Code is increasingly being challenged over its association with the over-exploitation of water bodies for economic gain at the expense of human and environmental needs. Many analyses have examined how the Water Code affects water management but have paid less attention to water governance: the institutional structures, processes, and practices of decision-making around water. The aim of this paper is thus to analyse how the Water Code has configured governance, how this governance has shaped the social relations of control over water, and how these social relations of control over water configure the wider political-economic order of the country. While analyses of governance as the practice of regulation shed light on the nature and functioning of the Water Code, the paper argues that approaching governance as the process of regulation-making reveals the power relations embedded in the framework and how these have transformed water-society relations to privilege capitalist and elite power. Revealing the power relations in the design, implementation, and defence of the Water Code both discloses the limitations of existing proposals for change and indicates new avenues for hydrosocial transformation.
\end{abstract}




\section{Introducción}

Durante las últimas tres décadas, el Código de Aguas de Chile de 1981 ha atraído mucha atención, tanto internacional como nacional, debido a su organización en torno a los llamados principios "neoliberales". El Código de Aguas instituyó derechos de propiedad privada permanentes sobre el recurso hídrico, habilitó mecanismos de mercado para la asignación del agua, y delegó la regulación a las organizaciones de usuarios. Por lo tanto, con base en los principios de privatización, mercantilización y comercialización, el marco legal fue diseñado para aumentar simultáneamente el papel del sector privado y reducir el del Estado en la gestión del agua, con el fin de fomentar la inversión de los usuarios en infraestructura y obtener la eficiencia del empleo del recurso por alentar la asignación de agua escasa a los usos económicos más productivos.

En lugar de un proyecto ideológico, como se describe a veces, es importante reconocer los motivos políticos detrás del Código de Aguas. Este fue elaborado en ausencia de oposición política bajo el régimen militar del general Pinochet (1973-1990). El Código de Aguas refleja los intereses de un nexo de elite político-económica entre el régimen militar, los tecnócratas gubernamentales y los sectores industriales (Budds, 2013). Los principios del Código de Aguas sirvieron para asegurar el agua para las industrias de exportación basadas en los recursos naturales de Chile, como la minería y la agricultura de exportación, lo que garantizó el acceso a los derechos de agua permanentes, libres de impuestos y relativamente libres de regulación estatal. El éxito de tales industrias, a su vez, tenía el objetivo de fomentar crecimiento económico bajo el programa neoliberal, lo que crearía apoyo para el gobierno militar y abriría el camino para que los tecnócratas asumieran el gobierno una vez que el ejército dejara el Poder Ejecutivo. Estas disposiciones para los flujos de agua fueron fuertemente defendidas por los mismos intereses político-económicos en los debates públicos sobre la modificación del Código de Aguas entre 1992 y 2005 (Budds, 2013).

El Código de Aguas fue diseñado por abogados y economistas, en lugar de hidrólogos u ingenieros hidráulicos, y, a pesar de las moderadas reformas realizadas en 2005, omite muchos elementos de la gestión y la gobernanza del recurso hídrico que caracterizan la política global del agua contemporánea. Primero, considera el agua como un insumo para las actividades económicas sin contemplar sus funciones ecológicas, sociales y culturales. Segundo, trata el agua como homogénea e inerte, y descuida la dinámica material del recurso; por ejemplo, se tratan las aguas superficiales y subterráneas como recursos separados a pesar de sus interconexiones. Tercero, el Código de Aguas no permite la gestión integrada de los recursos hídricos ni adopta la cuenca hidrográfica como escala de gestión. Cuarto, restringe la gestión del agua a los usuarios del agua, a la Dirección General de Aguas y a los tribunales civiles, sin ninguna función para otras partes interesadas.

Sin embargo, el Código de Aguas es cada vez más cuestionado en la práctica. La modificación en el año 2005 buscó abordar los resultados perversos percibidos, en particular el acaparamiento y la especulación de derechos sin usarlos, mediante la introducción de un sistema de patentes por no uso. Se fortalecieron algunas disposiciones ecológicas, como la evaluación de la capacidad del acuífero en lugar de simplemente el flujo del pozo al asignar derechos de agua subterránea. Varios informes empíricos han demostrado que la característica más notable del modelo chileno, las transacciones de los derechos de agua (a diferencia de los volúmenes de agua), es apenas evidente en la práctica (Bauer, 1997; Budds, 2004; Prieto, 2015). Por otro lado, lo que también se destaca es la forma en que la casi ausencia de regulación ya sea por parte de los usuarios, del Estado, de los tribunales o de un organismo independiente conduce a un control deficiente e incluso al abuso del recurso, ya que muchos usuarios buscan maximizar las extracciones de agua fuera del mercado. Además, desde 2010, Chile ha experimentado una creciente escasez de agua y varias crisis de agua en diferentes partes del país en relación con la denominada "megasequía" (Boisier, Rondanelli, Garreaud \& Muñoz, 2016; Garreaud et al., 2017; Garreaud et al., 2020), contra las cuales el Código de Aguas ha sido en gran medida ineficaz (Budds, 2020). El resultado de estos dos aspectos ha sido un escrutinio amplio e intensivo de la sociedad civil y el público, que condujo a un nuevo conjunto de reformas propuestas en 2015, así como a intervenciones tanto de los usuarios como del Estado para aumentar la oferta ante la escasez, descuidando convenientemente los principios neoliberales (Budds, 2020).

Por esta razón, el Código de Aguas se encuentra en un momento crítico. Gran parte del trabajo académico existente sobre su naturaleza e implementación se ha centrado en la efectividad de sus principios neoliberales, sobre todo los derechos privados del agua y los mercados, en varias regiones y sectores, y desde la perspectiva de diferentes grupos sociales (Bauer, 1997, 1998; Budds, 2003, 2004, 2007, 2009b, 2010, 2012; Hadjigeorgalis, 2008; Prieto, 2015, 2016; Romano \& Leporati, 2002). Los arreglos institucionales del Código de Aguas han sido centrales en algunos de estos análisis, con 
especial atención a los roles respectivos del "Estado" y el "sector privado" o los "privados". Sin embargo, si bien se ha prestado mucha atención a la gestión del agua y sus resultados bajo el Código de Aguas, se ha mostrado relativamente poca atención a la naturaleza de la gobernanza del agua: las estructuras institucionales, los procesos y las prácticas de toma de decisiones en torno a los recursos hídricos y los servicios sanitarios, con énfasis en cómo se configuran entre diferentes actores y en múltiples escalas (Bakker, 2007; Bakker \& Morinville, 2013).

El objetivo de este artículo, por lo tanto, es analizar (i) cómo el Código de Aguas ha configurado la gobernanza, (ii) cómo esta gobernanza ha moldeado las relaciones sociales de control sobre el agua, y (iii) cómo estas relaciones sociales de control sobre el agua configuran el orden político-económico del país. El texto está estructurado de la siguiente manera. La próxima sección examina los enfoques teóricos de la gobernanza del agua y su relación con los principios neoliberales. El tercer apartado presenta brevemente las características principales del Código de Aguas, y revisa la literatura que ha examinado el modo de gobernanza del agua en virtud de éste. La cuarta sección analiza las diferentes perspectivas, distinguiendo entre un enfoque gerencial que examina la práctica de la regulación y una perspectiva crítica basada en el proceso de construcción de la regulación. El último apartado concluye con el argumento que las disposiciones del Código de Aguas han cambiado las relaciones sociales de control sobre el agua y la identidad de ésta para privilegiar la acumulación del capital y los grupos elites al costo del interés social del país.

\section{La gobernanza del agua}

Bakker \& Morinville (2013, p. 1) definen la gobernanza del agua como:

la gama de procesos políticos, organizativos y administrativos a través de los cuales se articulan los intereses de la comunidad, se incorporan sus aportes, se toman e implementan decisiones, y se aseguran la responsabilidad de los que toman las decisiones con respecto al desarrollo y gestión de recursos hídricos y prestación de servicios de agua ${ }^{1}$.

Si bien la gestión del agua se refiere a las herramientas técnicas a través de las cuales se administran los recursos hídricos, la gobernanza del agua se refiere a las organizaciones e instituciones a través de las cuales se toman decisiones con respecto a los recursos hídricos. El cambio discursivo de la gestión a la gobernanza refleja la conciencia de que el agua no es simplemente un desafío técnico que se debe abordar por los hidrólogos e ingenieros hidráulicos, sino también una cuestión política que requiere la participación de una gama más amplia de actores sociales (Conca, 2006). En otras palabras, el agua es "asunto de todos" (Cosgrove \& Rijsberman, 2000).

Algunos autores conceptualizan la gobernanza del agua como la práctica de la regulación. Esto refleja el cambio del gobierno a la gobernanza en la ciencia política, a través del reconocimiento de que múltiples actores sociales más allá de las agencias estatales, tanto formales como informales, desempeñan roles en la definición y configuración de las normas, procesos, estructuras y organizaciones de toma de decisiones. La literatura que sigue esta tradición, por lo tanto, presta mucha atención a las preguntas pragmáticas de cómo se construyen e implementan los marcos institucionales del agua, lo que destaca los principios, reglas y organizaciones, a través de los cuales se toman decisiones sobre el agua (Conca, 2006). Una parte de esta literatura promueve la noción de "buen gobierno", que se refiere a aquello que es "participativo, orientado al consenso, responsable, transparente, receptivo, efectivo y eficiente, equitativo e inclusivo, y conforme a la ley" (UNESCAP, 2011), como la ruta principal para lograr una gobernanza del agua efectiva, eficiente y equitativa.

Sin embargo, existe el riesgo de que los principios de "buen gobierno" funcionen instrumentalmente, lo que lleva a Bakker \& Morinville (2013) a advertir contra su adopción como una "receta" para mejorar la toma de decisiones en torno al agua. Muchos defensores del buen gobierno presentan sus principios como soluciones automáticas con poca consideración de sus posibles barreras en la práctica: por ejemplo, los marcos legales requieren una aplicación efectiva, el acceso al recurso legal depende de los recursos y el estatus social, y la interpretación de la información requiere habilidades educativas. Esto se debe a que hay poco reconocimiento en estas versiones de cómo las relaciones de poder y la política pueden contribuir tanto a los desafíos del agua (Loftus, 2015; Swyngedouw, 2013), como a dar forma a la gobernanza para apoyar la gestión de ésta.

Otros autores han replanteado la gobernanza del agua como un proceso de construcción de la regulación, al referirse al papel del poder y del control en la gobernanza, y su contribución al orden político-económico (Bridge

${ }^{1}$ Todas las traducciones del inglés son propias de la autora. 
\& Perreault, 2009; Himley, 2008; Lemos \& Agrawal, 2006). Estos autores destacan que la gobernanza no es un esfuerzo neutral, sino cargado de poder, lo que a su vez sugiere que la gobernanza no se trata solo de hacer reglas sino también de lograr el poder. De esta manera, la gobernanza no es solo un proceso para tomar decisiones, sino también el medio para producir sujetos con las características necesarias para la implementación efectiva de esas decisiones.

Esta perspectiva pone énfasis no en los procesos de toma de decisiones por sí mismo, sino en las relaciones embebidas en ellos. Estos autores sugieren que el objeto de la gobernanza no debe ser el agua en sí, sino la relación entre el agua y la sociedad (Jepson et al., 2017; Linton \& Budds, 2014; Loftus, 2015). Esto, entonces, implica un alejamiento analítico de los arreglos de gobernanza en torno al agua, hacia una atención más centrada en las relaciones entre el recurso hídrico y los actores sociales (relaciones hidrosociales) a diferentes escalas. Por ejemplo, la formación de nuevas organizaciones, espacios y escalas para la gobernanza del agua a menudo se presentan como esfuerzos políticos pragmáticos, pero pueden servir como medios para perpetuar el poder político y/o la acumulación de capital (Bridge \& Perreault, 2009).

El hecho de reorientar el enfoque de la gobernanza del agua, desde las prácticas de toma de decisiones hasta las relaciones hidrosociales, implica permitir que las personas y las organizaciones participen significativamente, no solo como usuarios del agua, sino también como actores políticos que buscan transformar las relaciones a partir de las cuales se producen y reproducen las desigualdades del agua. Esto no es simplemente una intervención en los debates políticos o un llamado a nuevas formas de distribuir el agua de manera que se adhieran a los principios de buen gobierno, sino más bien un llamado al cambio político que implica "abogar por una posición en la que todos puedan participar democráticamente en la producción, distribución e intercambio de agua" (Loftus, 2015, p. 5).

\section{La neoliberalización de la gobernanza del agua}

Desde la década de 1980, el sector del agua (servicios sanitarios, recursos hídricos y servicios ambientales) ha estado cada vez más sujeto a la aplicación de principios neoliberales. En términos generales, estos comprenden la transferencia de la gestión y el financiamiento del Estado al sector privado, y/o el cambio concomitante a instrumentos de mercado como mecanismos de asignación y uso, por lo que el Estado se convierte en un facilitador y supervisor en lugar de un proveedor directo (Loftus \& Budds, 2016).

La justificación de los principios privados y del mercado es que la propiedad privada y la gestión del agua promoverán ganancias de eficiencia, ya que los propietarios se esforzarán por maximizar la eficiencia para maximizar las ganancias. Estos procesos comprenden tanto cambios materiales, como la transferencia de recursos o servicios hídricos de la administración pública a la privada, y prácticas discursivas, como la redefinición del agua de un bien público a un bien económico (Bakker, 2002).

Gran parte de este debate se ha centrado en cómo clasificar el agua como un tipo distinto de "bien" en el lenguaje de la economía institucional, a fin de definir el tipo de arreglos institucionales que deberían seguir. Los Principios de Dublín de 1992 reforzaron este debate al reformular el agua como un "bien económico", que fue movilizado por las instituciones internacionales para justificar un cambio del tratamiento del agua como un servicio público a un servicio que debería funcionar sobre la base de la recuperación total de costos. A diferencia del suministro de agua entubada, que tiene algunas características de un bien público y un bien privado (Budds \& McGranahan, 2003), el agua cruda generalmente se ha administrado como un recurso común.

Como es difícil o imposible excluir o restringir a los usuarios que tienen la capacidad de acceder al recurso, especialmente, a través de la infraestructura (Roberts \& Emel, 1992), el agua ha sido objeto de una sobreexplotación por parte de los usuarios individuales, un dilema reflejado en la teoría de Hardin (1968) de la tragedia de los comunes. Mientras Hardin (1968) abogó por la definición de los derechos de propiedad privada del agua para abordar este problema, Ostrom (1990) señaló que existen mecanismos relativamente sólidos de cooperación y gestión compartidos y/o comunitarios en muchas partes del mundo.

Sin embargo, la aplicación de los principios neoliberales a la gestión del agua ha vuelto a abogar por la creación de derechos de propiedad privada para los recursos previamente gobernados como recursos comunes. Esto, a su vez, significó que el debate ha vuelto a una clara distinción entre los sectores "público" y "privado". Los arreglos institucionales para el agua, ya sea que estén dirigidos por agencias estatales, particulares o la sociedad civil, marcan la diferencia en la forma en que se administran los recursos (gestión) y se toman las decisiones (gobernanza). Por ejemplo, una implicación común de transferir servicios de 
agua de una empresa de servicios públicos a una empresa privada es la pérdida de transparencia de la información debido a preocupaciones de sensibilidad comercial. Sin embargo, ambas opciones han sido criticadas porque ambas son insatisfactorias (control estatal o de mercado) y han excluido tradicionalmente a las comunidades (Bakker, 2007). Además, los sistemas privatizados y comercializados rara vez operan aislados del apoyo estatal (Harvey, 2003).

Con el fin de salvaguardar el interés público, generalmente se acuerda que se necesita una regulación efectiva, la que puede ser de una institución pública o independiente (Budds \& McGranahan, 2003). Si bien aquellos que apoyan los principios neoliberales también defienden la autorregulación por parte de la propia parte privada, en lugar del Estado específicamente, los críticos notan la tensión que se genera cuando la regulación no cabe con los intereses económicos de los propietarios de los recursos.

Por último, varios autores destacan las formas en que el contexto y la naturaleza del recurso afectan, y dan forma a los arreglos de gobernanza (Himley, 2008; Bakker, 2003, 2007). Como señala Bakker (2007), es particularmente difícil establecer los derechos de propiedad privada del agua, ya que es un recurso fluido y variable que no se limita o mide fácilmente, es un recurso parcialmente no sustituible que es esencial para las necesidades básicas, tiene importantes externalidades ambientales y de salud pública (cuyos costos son difíciles de calcular y reflejar en los mecanismos del mercado), y es un recurso altamente simbólico que tiene significados particulares y genera fuertes emociones.

\section{La gobernanza en el marco del Código de Aguas}

El Código de Aguas de 1981 define el agua como un "bien nacional de uso público". Se aplica a todas las fuentes de agua que cruzan los límites de la propiedad (principalmente ríos y acuíferos, para los cuales se necesitan derechos separados), y para todos los usos, excepto el agua potable rural (para humanos y ganado). No se estableció una priorización entre los usos, y no hubo obligación para los usuarios de usar su agua. El Código de Aguas permitió que los derechos de agua existentes se regularizaran como derechos de propiedad privada, y los desacoplaron de la tierra para permitir un mercado en el comercio del agua. Se podrían solicitar nuevos derechos de agua para fuentes no agotadas, las cuales, de estar disponibles, debían ser otorgadas por el Estado obligatoriamente, y de forma gratuita. Una vez que las fuentes se asignaron por completo, en principio se esperaba que la adquisición ocurriera en el mercado, ya que los derechos de agua se podían transferir (heredar, regalar, arrendar, vender) cuando se registraban correctamente en los registros de propiedades. Esto incluyó la medida en litros por segundo, pues el Código de Aguas convirtió las acciones de una fuente natural de agua (una medida proporcional), a litros por segundo (un volumen absoluto), con la intención de facilitar el comercio de derechos de agua al dejar en claro exactamente cuánta agua representaba un derecho (Bauer, 1997). Los derechos de agua privados estaban garantizados por la Constitución de 1980, lo que significa que solo podían ser expropiados por el Estado a precio de mercado.

En resumen, por lo tanto, el marco chileno se basa en tres principios. Primero, el uso del mercado para gestionar la demanda, mediante el cual se incentiva a los usuarios a comprar o vender derechos de agua de acuerdo con el valor del uso previsto. En segundo lugar, la designación de los derechos de agua como propiedad privada protegida por el Estado, que proporciona a los usuarios una seguridad de tenencia que se esperaba que fomentara la inversión de los usuarios en infraestructura de agua. Tercero, la delegación de regulación a las organizaciones usuarias de agua, la que considera evitar que la toma de decisiones sea discrecionales o politizadas. Esto, a su vez, está vinculado al principio neoliberal de que las decisiones deben ser tomadas por actores supuestamente neutrales y técnicos, en lugar de ser parciales y políticos (Budds, 2013).

En términos de gobernanza, el Código de Aguas implicaba una importante transferencia de control de los organismos estatales a los actores privados. La propiedad de los derechos de agua se transformó de concesiones estatales a derechos de propiedad privada, lo que significa que el Estado ya no tenía control sobre sus recursos hídricos (y por lo tanto sus cuencas, ecosistemas acuáticos y servicios ambientales). La propiedad también era individual, registrado al nombre de una persona natural o jurídica, lo que excluía la tenencia colectiva (que se refería especialmente a los grupos indígenas, hasta la promulgación de la Ley Indígena en 1993).

Los titulares de derechos de propiedad privada llegaban a determinar la asignación, el tipo de uso, y la medida en que se ejercía el agua, de acuerdo con sus intereses privados y económicos. Además, el papel de la Dirección General de Aguas (DGA) se redujo a la administración del sistema, principalmente la asignación de derechos de agua y la ejecución de estudios hidrológicos, aunque también podría hacerse cargo de la gestión del agua en caso de sequías oficialmente declaradas. Sin embargo, la DGA casi no jugaba ningún papel en la regulación, que 
en cambio se delegó a las organizaciones de usuarios de agua, principalmente a las asociaciones de canales de riego y a las Juntas de Vigilancia de un río (o sección de un río). Por ejemplo, eran solo los titulares de derechos de agua quienes podrían solicitar a la DGA que se investigue posibles violaciones de sus derechos de agua, o considerar la posibilidad de imponer restricciones a las fuentes de agua; la DGA no tenía mandato para estas funciones autónomamente (Budds, 2004). Los derechos de agua se regían por la ley civil (privada), y los conflictos debían resolverse entre los usuarios o los tribunales civiles, y no por la DGA. Otros interesados, que no son titulares de derechos de agua, no tienen ningún papel en la gestión del agua. De este modo, el Código de Aguas formó un marco regulatorio que benefició a los usuarios, al garantizar flujos de agua permanentes, libres de impuestos, y sin restricciones ni regulación.

La literatura existente que trata la gobernanza bajo el Código de Aguas se centra mayormente en los arreglos institucionales y sus implicaciones en la práctica. Si bien algunos análisis dan por sentado el marco al discutir los efectos, y desafíos de su implementación y aplicación, otros consideran estos resultados como producidos por los principios neoliberales que caracterizan el marco.

Algunos análisis se centran en las características institucionales neoliberales del marco, a saber, los derechos de propiedad privada, el comercio y la desregulación, y sus implicaciones para la gestión del agua, pero prestan relativamente poca atención a la toma de decisiones. Por ejemplo, Rogers \& Hall (2003, p. 30) describen al marco chileno como "un líder mundial en la gobernanza del agua", pero centran su discusión en los principios orientados al mercado para la gestión del agua. Estos autores señalan algunos de los desafíos del sistema, en particular con respecto a la participación de usuarios, la degradación de ecosistemas y los conflictos entre usos consuntivos y no consuntivos, pero los consideran como externalidades y no contemplan los grupos involucrados o la forma de tomar decisiones. Incluso algunos estudios que pretenden centrarse específicamente en la gobernanza, del mismo modo, casi no prestan atención a las estructuras, procesos y prácticas para la toma de decisiones en torno al agua. Por ejemplo, Valdés-Pineda et al. (2014) describen la administración y gestión del sistema del mercado del agua, y toman los principios privados y del mercado para constituir mecanismos de gobernanza en sí mismos, dado que los titulares de derechos de agua individuales deben ser los principales protagonistas de su uso.
Otros autores dan más atención a las funciones del Estado, los usuarios del agua y el sistema judicial dentro de un marco que combina los derechos de propiedad privada, las transferencias de mercado y la desregulación (Bauer, 2015; Budds, 2009a, 2012). El marco fomenta el control privado sobre el agua y significa que casi todas las decisiones sobre el uso del agua son tomadas por los titulares de derechos de agua. Por un lado, el Estado tiene una función administrativa en relación con la asignación de agua y la producción de información, visto que debe asignar los derechos de agua si están disponibles, y tiene un papel restringido en la evaluación de la capacidad de los cuerpos de agua para la asignación, y en la fiscalización del uso ilegal. De hecho, los casos en que la DGA ha ejercido cierta discreción a menudo se han enfrentado a desafíos legales (Budds, 2009a) o acciones de la Contraloría General (Bauer, 2015). Por otro lado, los titulares de derechos de agua pueden tenerlos sin obligación de usarlos, y pueden venderlos sin permiso o supervisión de terceros, lo que significa que el agua puede cambiar los usos de acuerdo con la demanda de las partes privadas. Por lo tanto, el marco privilegia los intereses privados sobre el interés público en relación con el uso del agua (especialmente la calidad ecológica y las necesidades humanas básicas), en el cual el sistema judicial parece ser la única institución que a veces sostiene el interés público (Bauer, 2015).

Se han planteado varios puntos como parte de los problemas y preocupaciones de gobernanza. Algunos autores sostienen que el Código de Aguas ha dado lugar a un modo muy centralizado de gobernanza del agua. Por ejemplo, Hurlbert \& Díaz (2013) afirman que la naturaleza centralizada del gobierno chileno restringe el papel de las agencias regionales de agua y los gobiernos locales en la gestión del agua, a pesar de que son responsables de algunos aspectos, como las respuestas a la sequía. Otros autores se refieren a funciones superpuestas de diferentes instituciones que se ocupan del agua, como entre la DGA y CONAF (Banco Mundial, 2011).

La OECD (2011) afirma que la gran cantidad de autoridades administrativas relacionadas con el agua en Chile dificulta que el país coordine el desarrollo, tanto horizontalmente (a través de instituciones y sectores) como verticalmente (a través de niveles y escalas de gobierno), lo que sugiere que puede explicar la autonomía disminuida de la DGA, un punto que también hacen Hearne \& Donoso (2014). Estos autores sugieren que la posición de la DGA es el resultado de un diseño institucional deficiente, mientras que Bauer (2015) afirma que la limitación del rol y de los recursos de la DGA es una decisión política para debilitar a la agencia. 
Varios autores señalan la naturaleza de arriba hacia abajo de la gobernanza del agua en Chile, y la falta de participación de los interesados en los problemas del agua, lo que atribuyen al hecho de que los titulares de derechos privados son los principales tomadores de decisiones (Bauer, 2004, 2015; Budds, 2009a; Reyes, Salas, Schwartz, Espinoza \& Morales, 2009). No existen foros formales para las interacciones entre las instituciones estatales y las organizaciones de la sociedad civil para comprometerse, y dar forma a las políticas y prácticas del agua. Irónicamente, esta ausencia de plataformas parece haber dado lugar a la creciente participación de los movimientos sociales que responden a la escasez de agua y la acumulación de derechos, ya que los principios neoliberales del Código de Aguas están cada vez más asociados con la sobreexplotación y la exclusión del agua (Bauer, 2015; Budds, 2020; Fragkou \& Budds, 2020).

Se hacen críticas similares sobre las formas en que la estructura y los procesos institucionales configuran la integración de diferentes fuentes, usos y escalas de agua. Algunos de los problemas más importantes del Código de Aguas, como su separación de las aguas superficiales y subterráneas, e institución de organizaciones de usuarios para secciones de ríos y acuíferos sin considerar sus conexiones (Bauer, 2004, 2015; Hearne \& Donoso 2014), fueron formulados en la década de $1980 \mathrm{y}$ desde entonces han quedado obsoletos en términos de gestión ambiental y del agua (Budds, 2009a). Esto impide la integración del agua a nivel de cuenca según la Gestión Integrada de Recursos Hídricos (GIRH), y se agrava por la ausencia de regulación (pública o independiente) para coordinar a los usuarios privados de agua (Bauer, 2004, 2015; Hearne \& Donoso, 2014). Mientras que algunos autores, como Bitran, Rivera \& Villena (2014), atribuyen esto a la rigidez de la gobernanza frente a condiciones cambiantes y/o nueva información, Bauer (2015) señala que las organizaciones de usuarios son tradicionalmente orientadas hacia la agricultura con poca capacidad para regular el uso noagrícola del agua, mientras que Budds (2013) enfatiza el debate polémico en torno a la modificación al Código de Aguas, a la que se opusieron los grupos conservadores. Varios autores señalan la falta de disposiciones para la resolución de conflictos, dado que estos son abordados por los propios usuarios, organizaciones de usuarios de agua o, en casos más complejos, el sistema judicial (los juzgados, los tribunales de apelación, e incluso la corte suprema) en lugar de la DGA (Bauer, 1997, 2015; Budds 2004; Rivera et al., 2016). Estos autores además agregan que existen problemas de confianza en el sistema judicial y la acción legal, que tanto tiene un costo prohibitivo (Budds, 2004) como está compuesto por jueces con poco conocimiento del Código de Aguas en general, y una renuencia a dictar sentencias que se podrían considerar como políticas (Bauer, 2004, 2015).

En un documento institucional, Vergara (2012) argumenta que el agua debe redefinirse como un recurso común que sea administrado colectivamente por los titulares privados de derechos de agua y las organizaciones de usuarios del agua $^{2}$. Este autor basa su argumento en su interpretación de los recursos comunes y sus formas de gobernanza. Intenta redefinir el agua en Chile como un recurso común, en lugar de un "bien nacional" o un bien público. Vergara (2012) sostiene que ni el Estado ni el "mercado" (o sea, el sector privado) han logrado un "uso productivo y positivo" (p. 1) del agua, y que "la realidad muestra" (pp. 4, 8) -sin presentar ninguna evidencia empírica- de que el agua es de hecho un recurso común que se autogobierna por los usuarios y sus organizaciones. Esto le lleva a sugerir un tercer modo de regulación: tratar las aguas como "bienes comunes gestionados colectiva y comunalmente por sus usuarios" (p. 9), pero mediante el cual los usuarios conservan sus derechos de propiedad privada de agua, y la DGA conserva una función administrativa.

El argumento de Vergara (2012) apoya los principios neoliberales subyacentes del Código de Aguas. Primero, afirma que el agua es inherentemente escasa y económicamente valiosa, principios utilizados para justificar los derechos de propiedad privada y la comercialización. Segundo, adopta una lógica productivista hacia el agua, afirmando que su función principal es ser explotada con fines económicos, en lugar de, por ejemplo, las necesidades básicas o la equidad social. Tercero, se refiere a la regulación en términos estrictos de protección de los derechos de propiedad privada, afirmando que su objetivo es la gestión eficiente y la prevención de conflictos "en la obtención y ejercicio de los derechos de agua" (p. 2). Cuarto, establece una clara distinción entre el "Estado" y el "mercado", este último definido en términos de la "autónoma decisión de los particulares" (p. 1), que refleja la libertad individual. Quinto, y de manera reveladora, se refiere a la "discrecionalidad excesiva" (p. 2) de la DGA, y enfatiza la necesidad de que esta agencia sea técnica y no política.

Vergara (2012) promueve su idea de "autogestión de un recurso común" (p. 3) para legitimar y defender el marco existente de derechos de propiedad privada y

${ }^{2}$ Véase también una crítica de Bauer (2015). 
ausencia de intervención estatal, especialmente frente a las crecientes demandas de los movimientos sociales. Su argumento refuerza la autorregulación de los usuarios y sus instituciones, que ya ocurre en el marco del Código de Aguas, sin especificar ninguna diferencia: las organizaciones de usuarios deben supervisar la distribución del agua según los derechos de cada usuario, los titulares de los derechos deben ejercer sus derechos según sus intereses particulares, y la DGA debe administrar el sistema, reiterando que "ya nada o muy poco tiene que hacer la DGA en esa cuenca o acuífero en que el reparto de las aguas comunes se está autogestionando por las juntas y demás organizaciones de usuarios" (p. 8) una vez que todos los derechos posibles se han asignado o regularizado. La única diferencia es la propuesta de un mecanismo mejorado para la resolución de conflictos, que podría ser un tribunal de agua o un panel de expertos técnicos.

El discurso de Vergara (2012) de considerar la práctica actual bajo el Código de Aguas como gobernanza de los recursos comunes, en lugar de la privatización y comercialización del agua, es erróneo y distractor. Es erróneo porque, si bien las fuentes de agua son recursos comunes porque son no o parcialmente divisibles, se gestionan a través de derechos privados en vez de colectivamente. Si bien este autor habla de gobernanza -un término que intercambia con "gestión" y "administración"- no hay espacio para que los usuarios o las organizaciones de usuarios tomen decisiones sobre cómo se gestionará y se compartirá el recurso, porque el Código de Aguas prescribe el modo de gestión, que no puede ser influenciada ni modificada. No se trata de la gestión de un recurso común, sino de la autorregulación de un bien privado. Es distractor porque busca cooptar el discurso de la sociedad civil de que el agua debe convertirse de un bien privado en un bien común, y potencialmente debilitarlo al afirmar que el Código de Aguas ya constituye un sistema de gestión de propiedad común.

Además, este autor no aborda las limitaciones importantes del Código de Aguas. Su análisis es teórico e ignora la práctica; por ejemplo, las organizaciones de usuarios no se han siempre constituidos, y a veces fueron impuestas por el Estado (Budds, 2020). Segundo, hay conflictos entre diferentes organizaciones de usuarios de agua, especialmente para las Juntas de Vigilancia en diferentes secciones de un río. Tercero, la gestión de las aguas superficiales y subterráneas tiene una dinámica muy diferente, la anterior es mayormente colectiva, en cuanto la última es en gran medida individualizada. Cuarto, los titulares de derechos de agua no siempre son usuarios (pueden ser especuladores), mientras que los usuarios de facto pueden no ser titulares de derechos de agua (pueden ser usuarios ilegales). Quinto, perpetúa el mismo modo de privilegiar a los titulares de derechos de agua y sus asociaciones para usar el agua de acuerdo con sus propios intereses, sin una participación más amplia de los interesados en las cuestiones de los recursos hídricos, para velar por el interés público. Los desafíos claves -el uso ilegal, la sobreexplotación de las fuentes, la degradación del medio ambiente, y la protección del agua potable rural- no se abordan en su texto.

\section{Discusión}

La mayoría de los análisis existentes se centran en el diseño y el funcionamiento de las características claves del marco (derechos de propiedad privada, transacciones y desregulación / autorregulación) y sus implicancias y resultados en la práctica. Como se señaló anteriormente, es posible distinguir entre las posiciones que tratan estos efectos como externalidades del Código de Aguas, las cuales podrían abordarse a través de refinamientos de la legislación, y aquellos que los posicionan como estructuralmente producidos por sus principios neoliberales, lo que sugeriría una reforma más profunda.

Sin embargo, muchas de estos análisis se refieren a cuestiones de gobernanza sin definir el término o establecer una base teórica explícita. Por ejemplo, Bauer (2015, p. 149) define la gobernanza como simplemente el "proceso de gobierno", y se refiere a "los procesos e instituciones legales y políticas para resolver conflictos sobre el agua" por varios grupos sociales más allá del Estado. Considerar la gobernanza como una simple toma de decisiones refleja un enfoque de la gobernanza como la práctica de la regulación, cuyo objetivo es la administración efectiva de los recursos hídricos. Si bien Bauer (2015) considera la política histórica y contemporánea del Código de Aguas, su atención se centra en la naturaleza y el funcionamiento de las reglas. Aunque considera las dimensiones políticas, como por ejemplo los debates políticos sobre la modificación del Código de Aguas, una limitación de este enfoque es que descuida los cambios más amplios y profundos que se aprovechan, a través del marco del Código de Aguas. En otras palabras, las relaciones políticas se posicionan en torno a las reglas y son externas a los recursos hídricos.

Por ejemplo, estos análisis tienden a centrarse en el papel de los diferentes partidos interesados y, en particular, establecen una distinción clara entre el Estado y el sector privado. Esto sirve para minimizar el papel que desempeñan los actores estatales en el apoyo y el beneficio del marco regulador del agua, por ejemplo, a través de las medidas que toman para protegerlo (Fragkou \& Budds, 2020). 
También se descuidan las identidades duales de los representantes del gobierno que también son usuarios del agua, y las formas en que la DGA puede rescatar el poder mediante la realización de evaluaciones hidrogeológicas (Budds, 2009a).

Además, al examinar las reglas y cómo funcionan, se corre el riesgo de ignorar las relaciones de poder que sustentan el diseño del Código de Aguas, así como las que se aprovechan a través de su operación (Budds, 2013). Abordar la gobernanza como un proceso de construcción de la regulación considera que la gobernanza no es un fin en sí mismo, sino un medio de organización social a través de la elaboración de normas en torno al agua, es decir, una relación interna. El foco se convierte entonces en cómo las disposiciones neoliberales del agua del Código de Aguas reconfiguran las relaciones agua-sociedad y con qué efectos.

En primer lugar, el Código de Aguas no fue originalmente un marco pragmático para lograr el uso óptimo del agua de acuerdo con la ideología neoliberal. Se creó para apuntalar la propiedad privada y la inversión en los principales sectores económicos de exportación de recursos naturales, con el fin de otorgar recursos hídricos al sector privado para el crecimiento económico de un modo permanente, libre de impuestos, sin restricciones y desregulado, y de una manera que fue difícil para los gobiernos posteriores modificar o revertir (Bauer, 1997; Budds, 2013). Como se mencionó anteriormente, los intereses creados en el Código de Aguas se hicieron evidentes en el debate público sobre la modificación del Código de Aguas, que no se trataba de la mejor manera de administrar el agua, sino de cómo preservar los principios del Código de Aguas, a saber, privado propiedad y libertad individual (Budds, 2013). Este análisis no solo se refiere a cómo los principios neoliberales dan forma al agua, sino a cómo la re-regulación del agua fue crucial para el funcionamiento del programa neoliberal (Budds, 2013).

En segundo lugar, los principios neoliberales del Código de Aguas no solo han cambiado las relaciones sociales de control sobre el agua, sino también la identidad tanto del agua como de los usuarios. La privatización y la mercantilización de los derechos de agua han inducido a la competencia por el agua e incentivado la sobreexplotación de las fuentes, para beneficios privados en lugar del interés público. El Código de Aguas también ha individualizado la propiedad y el uso del agua, como resultado de la concesión de derechos de agua individuales (en oposición a los colectivos), la conversión de los derechos a litros por segundo, y el uso cada vez más significativa de los pozos de agua subterránea, que han socavado la cooperación y el intercambio de agua en tiempos de escasez (Budds, 2020).

La regulación del agua principalmente por parte de particulares y en el interés privado, irónicamente, está demostrando de ser ineficiente e ineficaz para el crecimiento económico. Por ejemplo, el Estado ha tenido que gastar importantes recursos financieros para comprar agua de propietarios privados para abastecer a las comunidades rurales en tiempos de sequía, y la sobreexplotación de las fuentes de agua subterránea, incluso por extracciones ilegales, ha impactado los derechos de agua de las grandes industrias. Si bien el Estado ha respondido con proyectos de infraestructura liderados por la oferta, es probable que crear más oferta genere más demanda a largo plazo. Esta ineficiencia puede ser la ruina del Código de Aguas.

\section{Conclusiones}

El objetivo de este artículo fue analizar (i) cómo el Código de Aguas ha configurado la gobernanza, (ii) cómo esa gobernanza ha moldeado las relaciones sociales de control sobre el agua, y (iii) cómo estas dinámicas configuran el orden político-económico más amplio de Chile. Un análisis de la gobernanza como la práctica de la regulación arroja luz sobre la naturaleza y el funcionamiento de los principios neoliberales del Código de Aguas, y en particular sobre algunas de las limitaciones que surgen de los derechos de propiedad privada y la desregulación. Sin embargo, abordar la gobernanza como un proceso de construcción de la regulación dirige la atención hacia las relaciones de poder más amplias y profundas integradas en el Código de Aguas y cómo han transformado las relaciones agua-sociedad y la identidad de tanto el agua como los usuarios de agua en Chile. De esta manera, el documento mostró que el Código de Aguas fue diseñado a propósito para garantizar flujos de agua permanentes, libres, y desregulados a las principales industrias que generan crecimiento económico.

Si bien es importante ir más allá del binario públicoprivado que a menudo se invoca en relación con el Código de Aguas, este enfoque también destaca las limitaciones de tratar de definir el agua como un tipo particular de "bien". Esto es problemático, tanto porque los recursos hídricos y los derechos de agua no se ajustan exactamente a ninguna categoría, como también porque la naturaleza de la relación entre el agua y los ciudadanos debe ser primordial. Al revelar las relaciones de poder en el diseño, el funcionamiento y la defensa del Código de Aguas, un 
enfoque crítico hacia la gobernanza del agua revela las limitaciones de las propuestas de cambio existentes y abre nuevas ideas y vías para la transformación.

Estas vías deberían ir mucho más allá de la lista de aspectos generalizados para "mejorar", y las instituciones para "fortalecer", dentro del marco actual del Código de Aguas -pero sin ningún detalle sobre cómo se realizaríancomo lo sugiere el Banco Mundial (2011). También trascenderían los caminos más reflexivos y realistas de Bauer (2015), que han sido contemplados dentro del contexto político en Chile y la improbabilidad de no alcanzar ninguna reforma sustancial. Bauer propone (i) la redefinición de las obligaciones y restricciones sobre los derechos de agua para incorporar la noción de interés social, (ii) una mayor regulación estatal, (iii) una mejor capacitación de abogados y jueces, y (iv) una mayor participación de los interesados. Sin embargo, al mismo tiempo, las relaciones de poder embebidos en el Código de Aguas, y el programa neoliberal de Chile en general, están siendo opuestos cada vez más fuertemente. La última década ha sido testigo de las protestas contra los principios y efectos del Código de Aguas, y la breve apertura de nuevos espacios a través de mesas redondas regionales a partir de 2014. Si bien estas oportunidades de participación aún son limitadas, se podría exigir un papel en la gobernanza del agua, no en base de replantear el agua como un recurso común, sino por demandas de gestión colectiva, en la que todos los ciudadanos tienen derecho a participar política y democráticamente en la producción y distribución del agua.

\section{Referencias}

Bakker, K. (2003). An Uncooperative Commodity: Privatizing Water in England and Wales. Oxford: Oxford University Press.

Bakker, K. (2002). From state to market?: Water mercantilización in Spain. Environment and Planning A, 34(5), 767-790. https://doi.org/10.1068/a3425

Bakker K. (2007). The "commons" versus the "commodity": Alter-globalization, anti-privatization and the human right to Water in the global South. Antipode, 39(3), 430-455. https://doi.org/10.1111/ j.1467-8330.2007.00534.x

Bakker, K. \& Morinville, C. (2013). The governance dimensions of water security: A review. Philosophical Transactions of the Royal Society, A, 371(2002), 20130116. https://doi.org/10.1098/rsta.2013.0116
Banco Mundial. (2011). Chile: Diagnóstico de la Gestión de los Recursos Hídricos. Recuperado de http://documents.worldbank.org/curated/ en/452181468216298391/Chile-Diagn-243-stico-dela-gesti-243-n-de-los-recursos-h-237-dricos

Bauer, C. (1997). Bringing water markets down to earth: the political economy of water rights in Chile, 197695. World Development, 25(5), 639-656. https://doi. org/10.1016/S0305-750X(96)00128-3

Bauer, C. (1998). Slippery property rights: multiple water uses and the neoliberal model in Chile 1981-1995. Natural Resources Journal, 38(1), 109-155. Recuperado de https://www.jstor.org/stable/24888446

Bauer, C. (2004). Results of Chilean water markets: Empirical research since 1990. Water Resources Research, 40(9), W09S06. https://doi. org/10.1029/2003WR002838

Bauer, C. (2015). Water conflicts and entrenched governance problems in Chile's market model. Water Alternatives, 8 (2), 147-172. Recuperado de http://www.water-alternatives.org/index.php/alldoc/ articles/vol8/v8issue2/285-a8-2-8/file

Bitran, E., Rivera, P. \& Villena, M. (2014). Water management problems in the Copiapó Basin, Chile: Markets, severe scarcity, and the regulator. Water Policy, 16(5), 844-863. https://doi.org/10.2166/wp.2014.107

Boisier,J.P., Rondanelli,R., Garreaud,R.D.\&Muñoz,F.(2016). Anthropogenic and natural contributions to the Southeast Pacific precipitation decline and recent megadrought in central Chile. Geophysical Research Letters, 43(1), 413421. https://doi.org/10.1002/2015GL067265

Bridge, G. \& Perreault, T. (2009). Environmental governance. En N. Castree, D. Demeritt, D. Liverman \& B. Rhoads, Companion to Environmental Geography (pp. 475-497). Oxford: Wiley-Blackwell.

Budds, J. (2003). El acceso a los recursos de agua de los agricultores en el valle de La Ligua, Chile. Revista de Derecho Administrativo Económico, 2, 371-379. https://doi.org/10.7764/redae.11.10

Budds, J. (2004). Power, nature and neoliberalism: The political ecology of water in Chile. Singapore Journal of Tropical Geography, 25(3), 322-342. https://doi. org/10.1111/j.0129-7619.2004.00189.x 
Budds, J. (2007). Derechos de agua, pobreza y manejo ambiental en Chile: Recursos hídricos, minería y pueblos indígenas en el Norte Grande. En R. Boelens, A. Guevara-Gil, J. Hendriks \& J. Hoogesteger (Comps.), Pluralismo Legal, Reforma Hídrica y Políticas de Reconocimiento (pp. 157-174). Wageningen: WUR.

Budds, J. (2009a). Contested $\mathrm{H}_{2} \mathrm{O}$ : Science, policy and politics in water resources management in Chile. Geoforum, 40(3), 418-430. https://doi.org/10.1016/j. geoforum.2008.12.008

Budds, J. (2009b). The 1981 Water Code: The impacts of private tradable water rights on peasant and indigenous communities in Northern Chile. En W. Alexander (Ed.), Lost in the Long Transition: The Struggle for Social Justice in Neoliberal Chile (pp. 35-55). Lanham: Lexington Books.

Budds, J. (2010). Water rights, mining and indigenous groups in Chile's Atacama. En R. Boelens, D. Getches \& A. Guevara-Gil (Eds.), Out of the Mainstream: Water Rights, Politics and Identity (pp. 197-211). London: Earthscan.

Budds, J. (2012). La demanda, evaluación y asignación del agua en el contexto de escasez: Un análisis del ciclo hidrosocial del valle del río Ligua, Chile. Revista de Geografía Norte Grande, 52(1), 167-184. http:// dx.doi.org/10.4067/S0718-34022012000200010

Budds, J. (2013). Water, power, and the production of neoliberalism in Chile, 1973-2005. Environment and Planning D, 31(2), 301-318. https://doi.org/10.1068/d9511

Budds, J. (2020). Securing the market: Water security and the internal contradictions of Chile's Water Code. Geoforum, 113, 165-175. https://doi.org/10.1016/j. geoforum.2018.09.027

Budds, J. \& McGranahan, G. (2003). Are the debates on water privatization missing the point? Experiences from Africa, Asia and Latin America. Environment and Urbanization, 15(2), 87-113. https://doi.org/10. $1177 / 095624780301500222$

Conca, K. (2006). Governing Water: Contentious Transnational Politics and Global Institution Building. Cambridge and London: The MIT Press.

Cosgrove, W. \& Rijsberman, F. (2000). World Water Vision: Making Water Everybody's Business. London: Earthscan.
Fragkou, M. \& Budds, J. (2020). Desalination and the disarticulation of water resources: Stabilising the neoliberal model in Chile. Transactions of the Institute of British Geographers, 45(2), 448-463. https://doi. org/10.1111/tran.12351

Garreaud, R. D., Alvarez-Garreton, C., Barichivich, J., Boisier, J. P., Christie, D., Galleguillos, M., LeQuesne, C., McPhee, J. \& Zambrano-Bigiarini, M. (2017). The 2010-2015 megadrought in central Chile: Impacts on regional hydroclimate and vegetation. Hydrology Earth System Sciences, 21(12), 63076327. https://doi.org/10.5194/hess-21-6307-2017

Garreaud, R. D., Boisier, J. P., Rondanelli, R., Montecinos, A., Sepúlveda, H. H., \& Veloso-Aguila, D. (2020). The Central Chile Mega Drought (20102018): A climate dynamics perspective. International Journal of Climatology, 40(1), 421-439. https://doi. org/10.1002/joc.6219

Hardin, G. (1968). The tragedy of the commons. Science, 162(3859), 1243-48. https://doi.org/10.1126/ science.162.3859.1243

Harvey, D. (2003). The New Imperialism. Oxford: Blackwell.

Hearne, R. \& Donoso, G. (2014). Water markets in Chile: Are they meeting needs? En K. Easter \& H. Qiuqiong, Water Markets for the 21st Century (pp. 103-126). Dordrecht: Springer.

Hadjigeorgalis, E. (2008). Distributional impacts of water markets on small farmers: Is there a safety net? Water Resources Research, 44(10), W10416. https:// doi.org/10.1029/2007WR006527

Himley, M. (2008). Geographies of environmental governance: The nexus of nature and neoliberalism. Geography Compass, 2(2), 433-451. https://doi. org/10.1111/j.1749-8198.2008.00094.x

Hurlbert, M.A. \& Diaz, H. (2013). Water governance in Chile and Canada: A comparison of adaptive characteristics. Ecology and Society, 18(4), 61. http:// dx.doi.org/10.5751/ES-06148-180461

Jepson, W., Budds, J., Eichelberger, L., Harris, L., Norman, E., O’Reilly, K., Pearson, A., Shah, S., Shinn, J., Staddon, C., Stoler, J., Wutich, A. \& Young, S. (2017). Advancing human capabilities for water 
security: A relational approach. Water Security, 1 (1), 46-52. https://doi.org/10.1016/j.wasec.2017.07.001

Lemos, M.C. \& Agrawal, A. (2006). Environmental governance. Annual Review of Environment and Resources, 31, 297-325. https://doi.org/10.1146/ annurev.energy.31.042605.135621

Linton, J. \& Budds, J. (2014). The hydrosocial cycle: Defining and mobilizing a relational-dialectical approach to water. Geoforum, 57, 170-180. https:// doi.org/10.1016/j.geoforum.2013.10.008

Loftus, A. (2015). Water (in) security: Securing the right to water. The Geographical Journal, 181(4), 350356. https://doi.org/10.1111/geoj.12079

Loftus, A. \& Budds, J. (2016). Neoliberalizing water. En S. Springer, K. Birch \& J. MacLeavy, The Handbook of Neoliberalism. London: Routledge.

OECD. (2011). Water Governance in OECD Countries: A Multilevel Approach. Recuperado de http://www. oecd.org/cfe/regional-policy/watergovernanceinoecd countriesamulti-levelapproach.htm

Ostrom, E. (1990). Governing the Commons: The Evolution of Institutions for Collective Action. Cambridge: Cambridge University Press.

Prieto, M. (2015). Privatizing water in the Chilean Andes: The case of Las Vegas de Chui-Chiu. Mountain Research and Development, 35(3), 220-229. https:// doi.org/10.1659/MRD-JOURNAL-D-14-00033.1

Prieto, M. (2016). Bringing water markets down to Chile's Atacama Desert. Water International, 41(2), 191-212. https://doi.org/10.1080/02508060.2015.1107400

Roberts, R. \& Emel, J. (1992). Uneven development and the tragedy of the commons: Competing images for nature-society analysis. Economic Geography, 68(3), 249-71. https://doi.org/10.2307/144185

Romano, D. \& Leporati, M. (2002). The distributive impact of the water market in Chile: A case study in Limarí Province, 1981-1997. Quarterly Journal of International Agriculture, 41, 41-58. Recuperado de https://www.issuelab.org/resources/23391/23391.pdf

Reyes, B., Salas, S., Schwartz, E., Espinoza, E. \& Morales, L. (2009). Chile water governance assessment: final report. Unit 1E. Report for the Institutional Adaptation to Climate Change Project Committee, La Serena, Chile. Recuperado de http:// www.parc.ca/mcri/pdfs/papers/gov03.pdf

Rivera, D., Godoy-Faúundez, A., Lillo, M., Alvez, A., Delgado, V., Gonzalo-Martín, C., Menasalvas, E., Costumero, R. \& García-Pedrero, A. (2016). Legal disputes as a proxy for regional conflicts over water rights in Chile. Journal of Hydrology, 535, 36-45. https://doi.org/10.1016/j.jhydrol.2016.01.057

Rogers, P. \& Hall, A. (2003). Effective water governance. Global Water Partnership, Technical Background Paper. Stockholm: GWP. Recuperado de https://www.gwp.org/globalassets/global/toolbox/ publications/background-papers/07-effective-watergovernance-2003-english.pdf

Swyngedouw, E. (2013). UN Water Report 2012: Depoliticizing water. Development and Change, 44(3), 823-835. https://doi.org/10.1111/dech.12033

UNESCAP. (2011). What is good governance? Recuperado de http://www.unescap.org/pdd/prs/ ProjectActivities/Ongoing/gg/governance.asp

Valdés-Pineda, R., Pizarro, R., García-Chevesich, P., Valdés, J.B., Olivares, C., Vera, M., Balocchi, F., Pérez, F., Vallejos, C., Fuentes, R., Abarza, A. \& Helwig B. (2014). Water governance in Chile: Availability, management and climate change. Journal of Hydrology, 519, 2538-2567. https://doi. org/10.1016/j.jhydrol.2014.04.016

Vergara, A. (2012). Focalizando la agenda de un recurso común: El desafio de potenciar la autogestión de las aguas en Chile. Pontificia Universidad Católica de Chile. Recuperado de https://politicaspublicas. uc.cl/publicacion/serie-temas-de-la-agenda/serie-no56-focalizando-la-agenda-de-un-recurso-comun-eldesafio-de-potenciar-la-autogestion-de-las-aguas-enchile/

\section{Agradecimientos}

Agradezco a Michael Lukas y a Michael Handke por su trabajo en organizar este número especial sobre gobernanza, y estoy grata a los revisores de Investigaciones Geográficas para sus sugerencias pertinentes y útiles. 